\title{
Transcriptional control of toxT, a regulatory gene in the ToxR regulon of Vibrio cholerae
}

\author{
Darren E. Higgins ${ }^{1}$ and Victor J. DiRita ${ }^{1,2 *}$ \\ ${ }^{1}$ Department of Microbiology and Immunology, and \\ ${ }^{2}$ Unit for Laboratory Animal Medicine, University of \\ Michigan Medical School, Ann Arbor, Michigan 48109, \\ USA.
}

\section{Summary}

Co-ordinate expression of many virulence genes in Vibrio cholerae is under the control of the Toxh and ToxT proteins. These proteins function in a regulatory cascade in which ToxR is required to activate toxT, and ToxT activates virulence genes. The precise mechanism for ToxR activation of toxT is unknown, but data presented in this report suggest a direct involvement of ToxR. Primer extension and gene fusion analyses identified a ToxR-regulated promoter directly upstream of toxT, immediately following a region of inverted repeats capable of terminating transcription. Gel mobility shift studies indicate that ToxR binds DNA within the inverted repeat region, yet preliminary evidence suggests that ToxR binding alone is not sufficient for activation of toxT. Possible mechanisms of ToxR-dependent toxT expression are discussed.

\section{Introduction}

Co-ordinate expression of many virulence genes in the human diarrhoeal pathogen Vibrio cholerae is under the control of the ToxR, ToxS and ToxT proteins (DiRita et al., 1991; Higgins et al., 1992; Miller et al., 1987; 1989; Miller and Mekalanos, 1984; Peterson and Mekalanos, 1988; Taylor et al., 1987). ToxR is an inner membrane protein that shares homology with members of the twocomponent family of transcriptional activators found in various species of bacteria (Miller et al., 1987; Stock et al., 1989). Unlike other members of this family, however, ToxR does not share the characteristic phosphoacceptor domain and is believed to be activated in some manner other than phosphorylation by a sensor kinase. It has been proposed that ToxR is active as a homodimer and that ToxS is required to stabilize ToxR into the dimer

Received 15 February, 1994; revised 23 May, 1994; accepted 10 June, 1994. *For correspondence. Tel. (313) 9363804; Fax (313) 9363235. form (DiRita and Mekalanos, 1991). The ToxR protein activates transcription of the genes coding for the cholera toxin (ctxAB) by directly binding to a repeated DNA element, TITTGAT, which is present in three to eight copies immediately upstream of the $c t x$ promoter (Miller et al., 1987). In addition to the $c t x$ genes, other genes necessary for colonization and full virulence require ToxR for their expression. These include genes for the production of the toxin-coregulated pilus (TCP) (Taylor et al., 1987), and several accessory colonization factor (ACF) genes (Peterson and Mekalanos, 1988; Parsot and Mekalanos, 1992). Furthermore, expression of these virulence genes is under environmental control, as changes in $\mathrm{pH}$, osmolarity and temperature during in vitro growth modulate expression of ToxR-dependent genes (DiRita et al., 1991).

Other than the ctx operon, ToxR does not directly activate transcription of the virulence genes described above (DiRita et al., 1991). Instead, ToxR mediates its control of these additional virulence genes through the ToxT protein (DiRita et al., 1991; Higgins et al., 1992). ToxT is a member of the AraC family of transcriptional activators (Higgins et al., 1992) and it activates many ToxR-regulated genes, including $t c p A, t c p C$, tcpl and ctxAB (DiRita et al., 1991). Previous work on the ToxR system led to a cascade model in which ToxR-regulated activation of toxT transcription results in co-ordinate activation of virulence genes by ToxT in $V$. cholerae (DiRita, 1992; Higgins et al., 1992). This model is supported by the observations that toxT specific message is undetectable in toxR mutant cells and that toxT under control of the constitutive tet promoter restores expression of TсpA and $\mathrm{Ctx}$ when introduced into a toxR mutant $V$. cholerae (DiRita et al., 1991; Higgins et al., 1992). Furthermore, in toxR mutant cells expressing toxT from the tet promoter, virulence gene activation is no longer subject to environmental modulation (DiRita et al., 1991). Therefore, the regulated expression of toxT is an important step in controlling virulence in $V$. cholerae. The precise role of ToxR in controlling expression of toxT is as yet uncharacterized. Whether ToxR directly activates toxT in a manner similar to its activation of $\operatorname{ctx} A B$, or does so indirectly through another mechanism is unknown. In this report, we present data that suggest a direct involvement of ToxR in toxT activation and characterize cis-acting regions involved in the regulated expression of toxT. 


\section{Results}

\section{Mapping of the toxT transcription initiation site}

The tox $T$ gene resides on the $V$. cholerae chromosome 210 nucleotides downstream of the $t c p F$ gene with its stop codon overlapping the start codon for the tcpJ gene (Fig. 1; Higgins et al., 1992; Kaufman et al., 1991; Ogierman and Manning, 1992). The repeated ToxRbinding motif TIITGAT, which is a component of the ctx promoter, is not present in the intergenic region between tcpF and toxT, or within several kilobases upstream of tcpF (Higgins et al., 1992; Ogierman et al., 1993). The tcpF-toxT intergenic region does contain several inverted repeat elements which have the potential to form secondary stem-loop structures and may play a role in the expression of toxT (see below). In order to define the initiation site of toxT transcription, primer extension mapping was performed using RNA isolated from $V$. cholerae. Wild-type (O395) and toxR mutant (JJM43) classical strains of $V$. cholerae were grown overnight at $30^{\circ} \mathrm{C}$ in Luria-Bertani (LB) medium with a starting $\mathrm{pH}$ of 8.5, a condition that does not favour ToxR-regulated gene expression (DiRita et al., 1991). These were then diluted (1:10) into fresh medium at $\mathrm{pH} 6.5$ and grown at $30^{\circ} \mathrm{C}$, conditions that favour ToxR-regulated gene expression, for one hour. RNA was then isolated from cultures and primer extension analysis performed using a primer complementary to the very 5 portion of the toxT open reading frame.

Two predominant primer extension products that mapped within the tcpF-toxT intergenic region were identified using RNA from the wild-type strain, whereas only one of these was identified when RNA from the toxR mutant was used (Fig. 2). The primer used in the extension analysis was used in a DNA sequencing reaction to determine the precise nucleotide to which each primer extension product maps (indicated by asterisks in Fig. 3). Previous computer analysis of the intergenic region identified a putative prokaryotic promoter element (Higgins et al., 1992) directly upstream of the site that maps closer to the start of the toxT gene (Fig. 3). The spacing between the site mapped by primer extension and this element is consistent with the element being a promoter for transcription initiation at the site. However, the -35 portion is not a good match with the consensus prokaryotic sequence (Fig. 3). Poor match to the

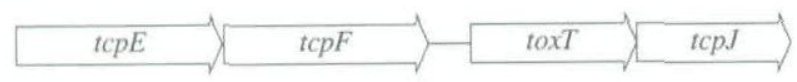

$\frac{2 \mathrm{~kb}}{2}$

Fig. 1. Genetic organization of a portion of the tcp region of $V$. cholerae. The organization of the tcpE, $F, J$ and toxT genes is shown. The arrows indicate the direction of transcription.

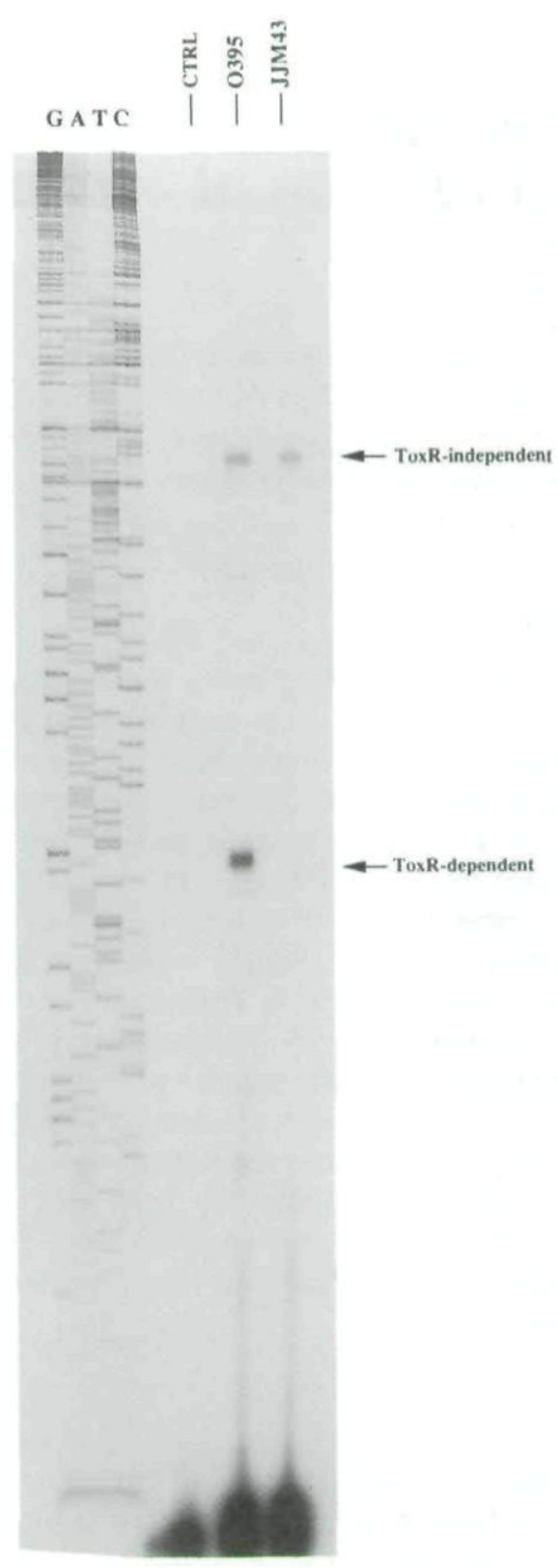

Fig. 2. Primer extension analysis of toxT transcripts. RNA was isolated from $V$. cholerae wild type (O395) or toxR mutant (JJM43) classical strains after $1 \mathrm{~h}$ of growth from shifting overnight cultures grown in LB medium at $\mathrm{pH} 8.5$ to $\mathrm{pH} 6.5$. A radiolabelled primer was incubated with the RNA, and reverse transcriptase was added to produce a DNA copy of the transcript, as described in the Experimental procedures. The control lane had no RNA added. The same primer was used to prime dideoxy sequencing products from a DNA template that contained the tcp region shown in Fig. 1. The letters above each lane indicate the dideoxynucleotide used to terminate each reaction. The ToxR-dependent and independent extension products are indicated.

consensus -35 element has been noted for promoters that require activators for elevated expression (Raibaud and Schwartz, 1984). Because the primer extension product mapping to this site was not identified using RNA 
from the tox $R$ mutant strain, we conclude that this is a potential ToxR-dependent transcription initiation site. The other primer extension product, which was detected using RNA from both wild-type and tox $R$ mutant strains is therefore ToxR-independent. The site to which this ToxRindependent product maps is within one of the inverted repeats noted above and is not preceded by a consensus promoter element directly upstream. Possible sources of this primer extension product will be discussed below, but from the data in Fig. 2, we conclude that a ToxRregulated promoter is present upstream of the tox $T$ gene. Additional analysis indicates that both of the primer extension products shown in Fig. 2 are specific for toxT mRNA, since primer extensions with different primers specific for various regions of toxT map to the same two sites (data not shown).

\section{Transcription termination within the tcpF-toxT intergenic region}

We previously speculated that tox $T$ expression could be the result of transcription initiating from a promoter upstream of $t c p F$, with the majority of transcripts terminating within the potential stem-loop structures downstream of tcp $F$ and a low percentage of the transcripts proceeding into toxT (Higgins et al., 1992). Although other investigators have indicated that a potential stem-loop structure following $t c p F$ would serve as a Rho-independent transcriptional terminator delineating the TCP gene cluster (Ogierman and Manning, 1992; Ogierman et al., 1993), experiments directly testing the ability of this region to terminate transcription have not been reported. To investigate whether DNA from the tcp $F$-tox $T$ intergenic region can terminate transcription, we used the transcription
Table 1. Transcription termination within the tcpF-toxT intergenic region.

\begin{tabular}{llc}
\hline & $\begin{array}{l}\text { Units of galactokinase } \\
\text { activity }^{\mathrm{b}}\end{array}$ & \% of control activity \\
\hline pKL600 (control) & 332 & 100 \\
pB51 & 61 & 18.4 \\
ptR2 & 27 & 8.1 \\
\hline
\end{tabular}

See Table 3 for description of the reporter plasmids.

a. Plasmid copy number was verified to be similar in all cases.

b. Numbers represent the average of at least three individual experiments.

c. Percent of control activity represents the amount of galactokinase activity relative to the pKL.600 reporter with no terminator present.

termination assay vector pKL600 (Cheng et al., 1991). pKL600 is a pBR322-based vector containing a plac promoter followed by a multiple cloning region preceding the gene coding for galactokinase (galK). A segment of the intergenic region (between the $(\wedge)$ markings shown in Fig. 3) was cloned into the multiple cloning region of pKL600 yielding plasmid pB51. This portion of the intergenic region was chosen because it contains the inverted repeats, but lacks the putative -10 element upstream of the ToxR-dependent transcription initiation site identified by primer extension. Transcription termination was assayed in Escherichia coli and was calculated by comparing the amount of galK activity in pB51 to that of the pKL600 vector containing no termination region. The strain with pB51 showed a decrease of at least $80 \%$ in the amount of galK activity as compared to that of pKL600 (Table 1). This indicates possible transcription termination within the intergenic region upstream of toxT. The data also suggest that some transcripts $(-20 \%)$ may read through to toxT. In a control experiment, a plasmid containing the phage lambda tR2 terminator, a strong terminator of
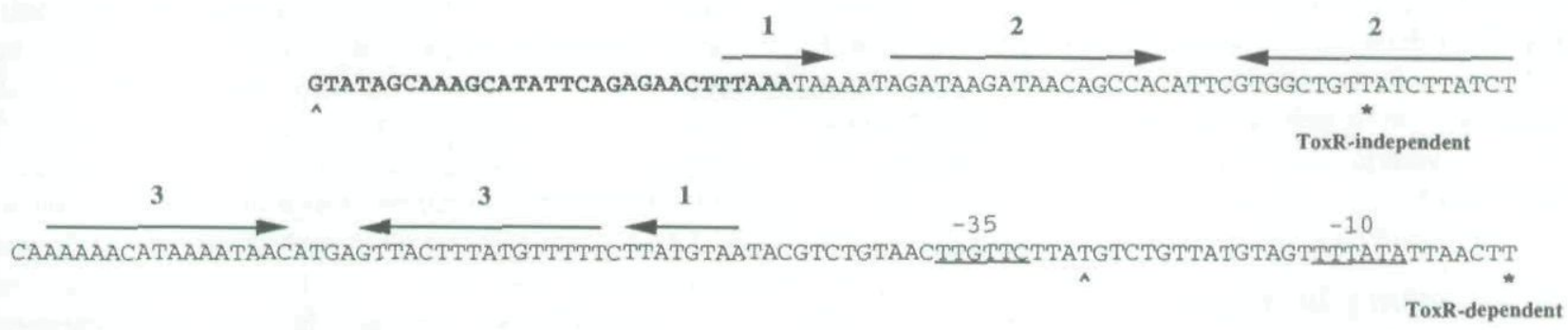

toxT

CGATTTTAAGATAACTTTACGTGGATGGCTCTCTGCGTTTATTTATATATCTTCAGAGTAGAACGCAATGATTGGGAAAATCTTPTCAAACTAATG

Extension Primer

Fig. 3. Nucleotide sequence of the tcpF-tox $T$ intergenic region. Sequences within $t c p F$ and tox $T$ are shown in boid. Arrows above the sequence indicate inverted repeat regions and are arbitrarily numbered as 1,2, and 3 . The putative Shine-Dalgamo (SD) translation initiation sequence and ToxR-dependent promoter sequence are underlined and labelled. The ToxR-dependent and -independent primer extension sites are labelled and indicated with asterisks ( $\left.{ }^{*}\right)$. The extension primer used was complementary in sequence to the very $5^{\prime}$ portion of toxT as indicated by the labelled arrow. Nucleotides within and including those underscored by a $(\wedge)$ represent the region tested for the ability to terminate transcription. 
Units of $B$-galactosidase

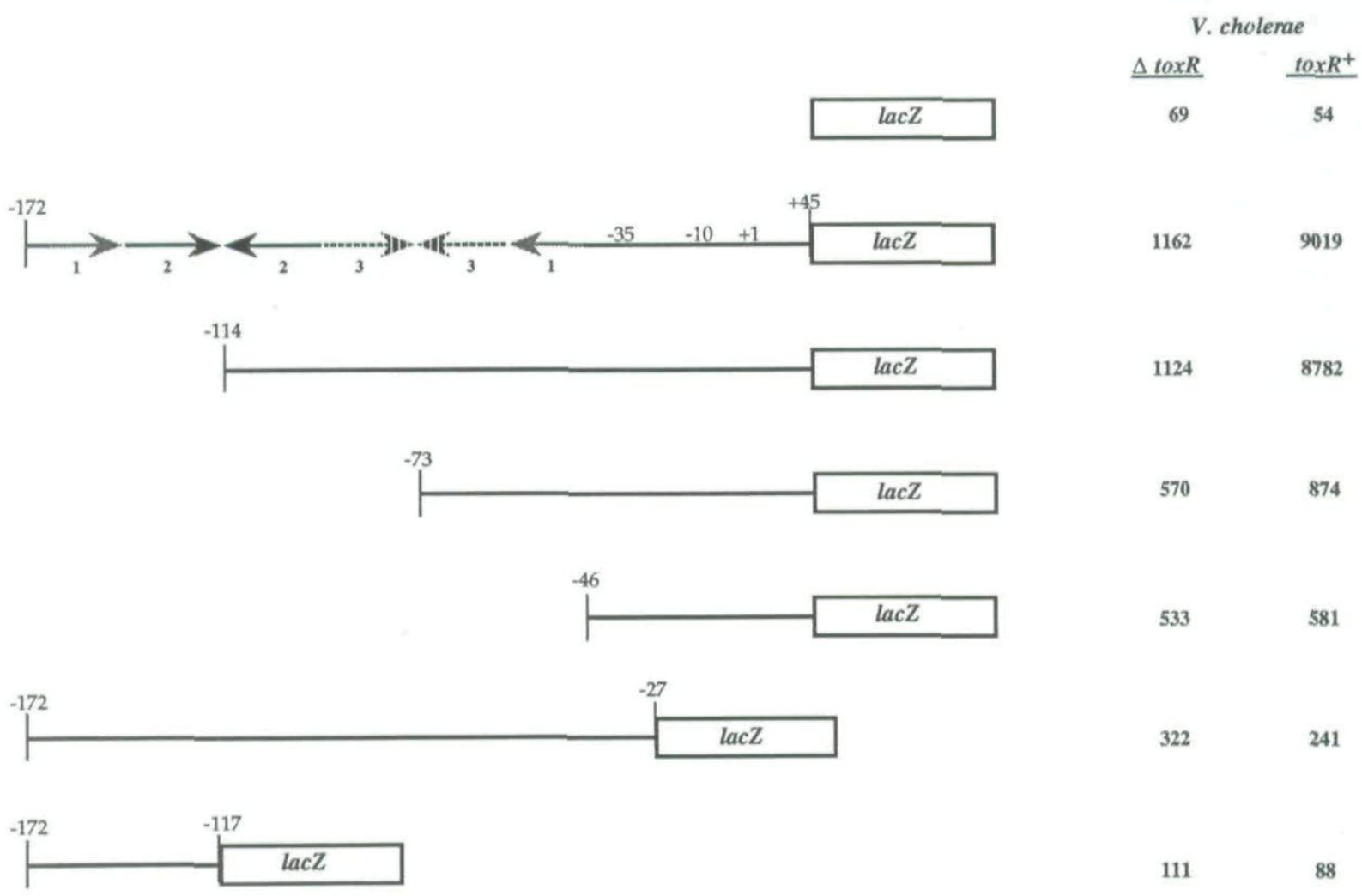

Fig. 4. Analysis of tcpF-toxT intergenic region fusions in V. cholerae. Fusions were made by cloning PCR products harbouring portions of the tcpF-toxT intergenic region in front of a promoterless lacZ gene on a broad-host-range plasmid. After determining that the PCR product sequence was wild type, the recombinant plasmids were introduced into $V$. cholerae. Beta-galactosidase activity was measured as described in the Experimental procedures and is reported in Miller units (Miller, 1972). $\Delta$ tox $R$ and tox $R^{+}$represent the results from fusions in V. cholerae strains JJM43 and O395, respectively. Arrows represent the presence of inverted repeats in the DNA sequence and are labelled as in Fig. 3. The ToxR-dependent primer extension product maps to the position labelled +1 on the figure and the positions of the putative -35 and -10 RNA polymerase binding elements are noted. Other numbers on the figure represent positions relative to the +1 . The $t c p F$ gene coding sequence ends within the upstream inverted repeat number 1.

transcription (Cheng et al., 1991), yielded only $8 \%$ of galK activity as compared with pKL600 (Table 1). Although we infer from these experiments that the cloned tcpF-toxT intergenic DNA contains a transcription termination signal, we cannot rule out the possibility that an RNA processing event is occurring which leads to a decrease in galK mRNA.

\section{Identification of a ToxR-regulated toxT promoter}

The primer extension analysis reported above identified a potential ToxR-dependent transcription initiation site directly upstream of toxT within the tcpF-toxT intergenic region. In order to localize the ToxR-dependent promoter responsible for toxT transcription, fusions were constructed by cloning fragments from the tcpF-toxT intergenic region generated by PCR in front of a promoterless lac Z gene on a broad-host-range plasmid (pTL61T; Linn and Pierre, 1990). The recombinant plasmids were introduced into the $V$. cholerae wild type (O395) and toxR mutant (JJM43) classical strains and $\beta$-galactosidase activity was measured (Fig. 4). A transcriptional fusion containing DNA spanning the end of the tcpF gene to just before the start of the toxT open reading frame $(-172$ to +45 relative to the ToxR-dependent start site) expressed eightfold more $\beta$-galactosidase activity in wild type than in the toxR mutant. A similar result was seen when DNA from -114 to +45 relative to the ToxR-dependent start site was fused to lacZ. However, ToxR-regulated $\beta$-galactosidase activity was essentially abolished when a fragment containing DNA from -73 to +45 relative to the start site was used. When a fragment missing the -10 portion of the putative promoter (containing DNA from -172 to -27 relative to the ToxRdependent start site) was fused to lac $Z$, relatively low $\beta$-galactosidase activity was expressed in both wild-type and toxR mutant strains of $V$. cholerae. Finally, fusion of DNA from -172 to -117 (relative to the start site) to 


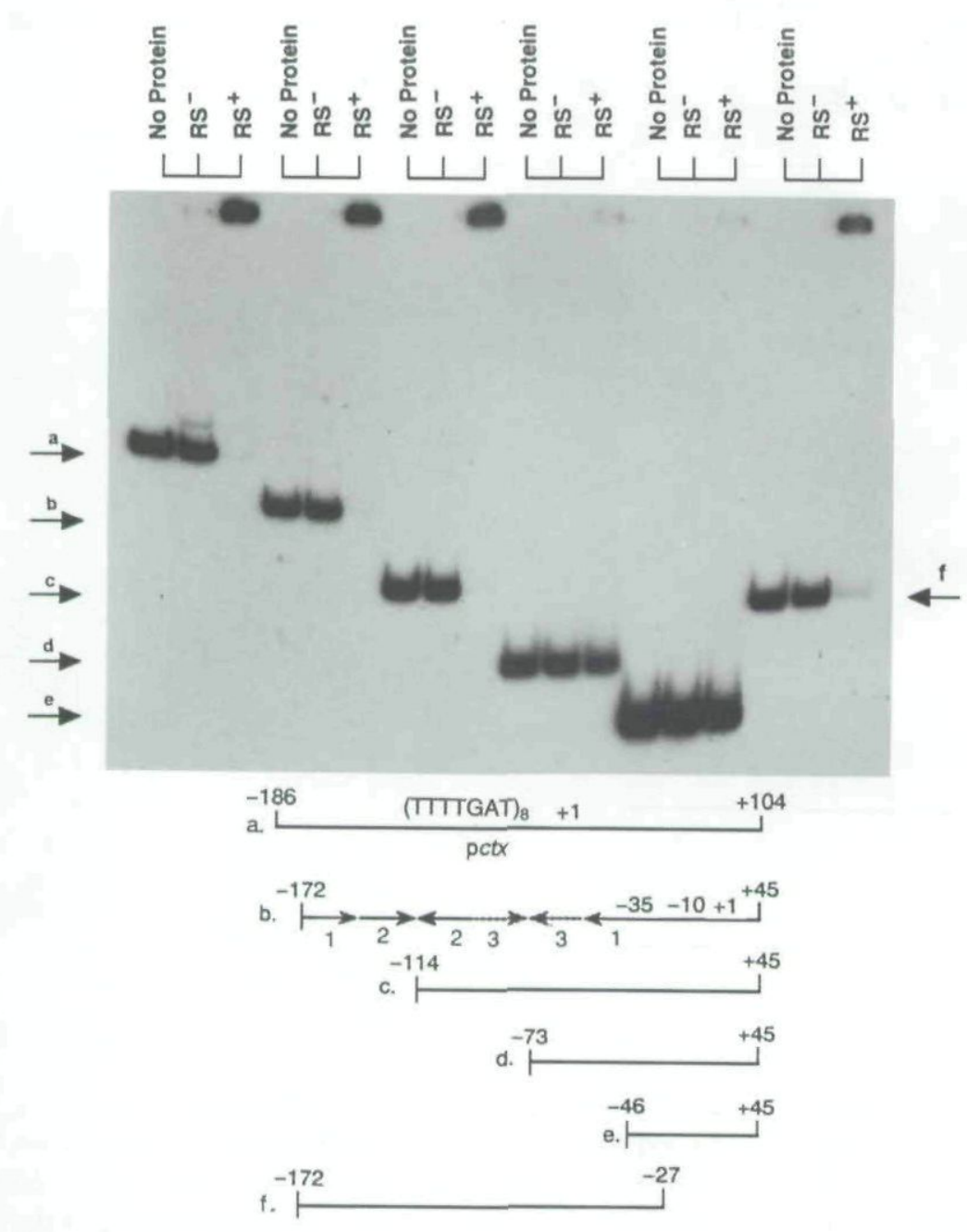

Fig. 5. Ability of ToxR to bind and shift toxT promoter DNA. PCR products representing the $c t x$ promoter region or various regions of the toxT promoter were radiolabelled and used in gel mobility-shift assays with ToxR as described in the Experimental procedures. Fragments are labelled $a-f$ and represent the corresponding DNA region depicted below the figure. The $c t x$ promoter fragment contained eight copies of the TITTGAT ToxR-binding sequence. Labels at the top of the figure indicate whether DNA alone (No Protein), membranes without ToxR and ToxS ( $\mathrm{RS}^{-}$), or membranes containing ToxR and ToxS ( $\mathrm{RS}^{+}$) were used in the assay. lac $Z$, resulted in a low level of $\beta$-galactosidase activity that was unaffected by ToxR.

Although conclusions regarding the magnitude of ToxRdependent activation of toxT expression cannot be drawn from these experiments because the reporter gene is on a plasmid, the results strongly suggest that a ToxRregulated promoter resides upstream of toxT. Hence, we conclude that the promoter element identified in Fig. 3 is required for directing toxT transcription initiation, presumably at the ToxR-dependent start site mapped by primer extension (Fig. 2), and that a region of DNA between -114 and -73 relative to the ToxR-dependent start site is necessary for ToxR-regulated activation of this promoter.

\section{ToxR binds to toxT promoter DNA}

ToxR binds to DNA containing at least three copies of the sequence TITTGAT upstream of the cholera toxin promoter and activates transcription of the cholera toxin genes (Miller et al., 1987). To determine whether or not ToxR-dependent activation of the toxT promoter involves direct interaction by ToxR, the ability of membranes containing ToxR to bind toxT promoter DNA was assayed in gel mobility-shift experiments (Miller et al., 1987; Ottemann et al., 1992). Total cell membranes were prepared from $E$. coli strain AAEC189 harbouring either the toxRS plasmid pVJ21 (Miller et al., 1989) or the vector for pVJ21, pACYC184 (Chang and Cohen, 1978). These membrane preparations were used in DNA gel mobility-shift assays with the PCR fragments used in the promoter studies described above. As shown in Fig. 5, membranes containing ToxR shifted fragments containing DNA from 114 to 172 nucleotides upstream of the ToxRdependent transcriptional start site. The fragments that are shifted correlate exactly with those that directed ToxR-regulated $\beta$-galactosidase activity in $V$. cholerae when fused to lacZ (Fig. 4). For example, a DNA fragment 


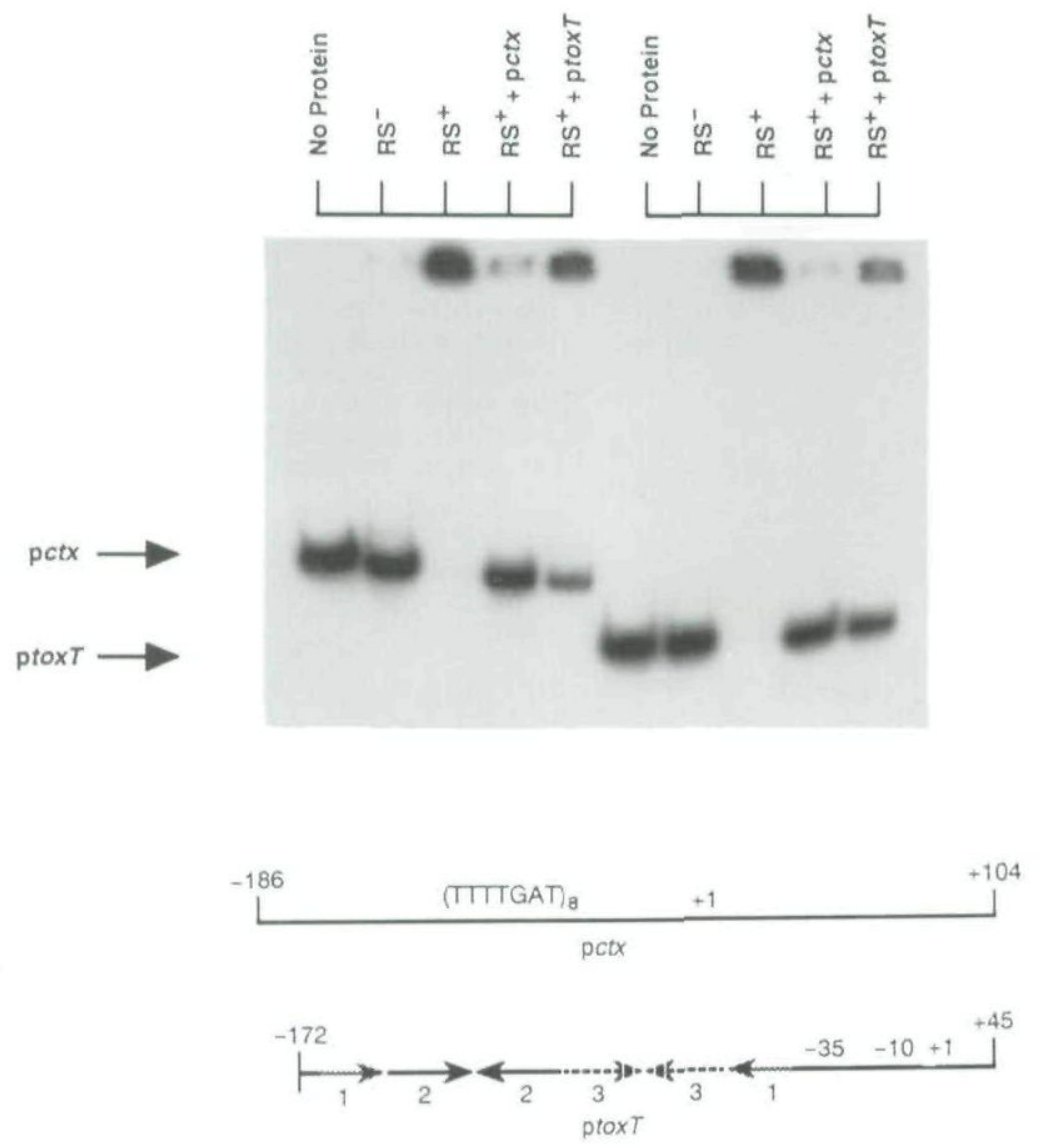

Fig. 6. Ability of toxT and ctx promoter DNA to compete for ToxR binding. Gel mobility-shift assays were performed as in Fig. 5. +pctx and + ptox $T$ represent assays in which an excess amount of unlabelled ctx or toxT promoter fragment was added to the binding reaction. with 114 nucleotides of upstream DNA had both ToxR-dependent promoter activity and was bound by membranes containing ToxR, while a fragment with 73 nucleotides of upstream DNA conferred very little ToxRdependent $\beta$-galactosidase activity and was not detectably bound by ToxR-containing membranes. Although the fragment missing the -10 portion of the ToxRdependent promoter did not exhibit any regulated activity (Fig. 4), it still contains the ToxR-binding region upstream of -73 and consequently was shifted in the binding assay ( $f$ in Fig. 5).

\section{ctx and toxT promoter DNA can compete for ToxR binding}

While the ToxR-binding region upstream of the ToxRdependent toxT promoter is rich in secondary structure, partially because of the presence of a potential transcription terminator, it does not contain the repeated TITTGAT motif required for ToxR binding to the ctx promoter. This suggests that ToxR can bind to at least two different DNA primary sequences. To determine if the $c t x$ and tox $T$ promoters could compete for ToxR binding, competition gel mobility-shift assays were performed.

Radiolabelled ctx or toxT promoter fragments were mixed with an excess of unlabelled fragment of the same or opposing promoter and used in the gel mobility-shift assay (Fig. 6). Excess unlabelled DNA containing the ctx or toxT ToxR-binding regions was able to compete for ToxR binding to labelled fragments containing either promoter. In addition, a DNA fragment of 243 nucleotides from the $V$. cholerae tcp gene cluster that does not have known ToxR-dependent promoter activity was not able to compete for binding to either the ctx or toxT promoter fragments (data not shown). Thus, ToxR appears to bind specifically to the two different promoters.

\section{ToxR does not directly activate the toxT promoter in E. coli}

ToxR binding upstream of the ctx promoter is sufficient to activate ctx transcription in E. coli (Miller et al., 1987). As ToxR can bind directly upstream of the toxT promoter, we wished to determine if ToxR could directly activate transcription of toxT. The same lac $Z$ fusion plasmids tested in $V$. cholerae were introduced by transformation into $E$. coli strain AAEC189, containing either the toxRS plasmid pVJ21 or vector plasmid pACYC184, and $\beta$-galactosidase activity was measured (Fig. 7). In the presence of ToxR, fusions harbouring the ToxR-binding portion of the toxT 
Units of $\beta$-galactosidase

E. coli

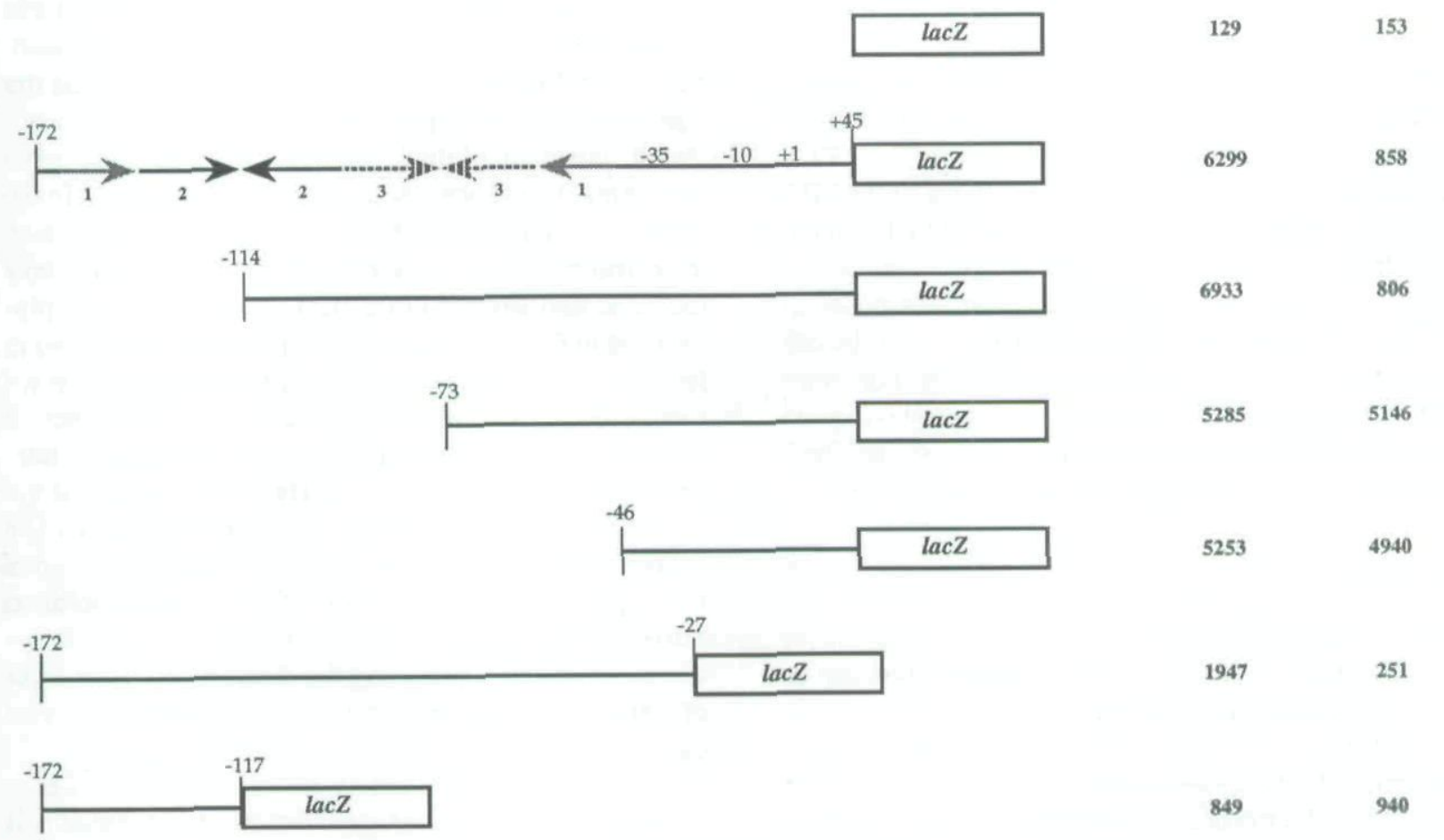

Fig. 7. Analysis of tcpF-toxT intergenic region fusions in E. coli. Fusions analysed were identical to those described in Fig. 4. -ptoxRS and +ptoxRS represent respectively, results from fusions in E. coli strain AAEC189 containing plasmid pACYC184 or pVJ21.

promoter (containing DNA from -172 to +45 or -114 to +45 relative to the ToxR-dependent start site) did not show any activation, but instead demonstrated an eightfold decrease in the amount of $\beta$-galactosidase activity. This inhibitory effect of ToxR was not observed when lac $Z$ fusions containing only 73 or 46 nucleotides of DNA from directly upstream of toxT were used; high levels of $\beta$-galactosidase activity were expressed from these fusions in both the absence and presence of ToxR. The decrease in $\beta$-galactosidase activity observed with the fragment lacking the putative -10 element (containing DNA from -172 to -27 relative to the ToxR-dependent start site) is consistent with our conclusion that the $\beta$-galactosidase activity from the fusions is predominantly due to initiation from the ToxR-dependent site shown in Fig. 3. However, this fragment does contain the ToxRbinding region and a similar repression of activity was seen when ToxR was present (Fig. 7). From these observations it appears that ToxR does not directly activate transcription of the toxT promoter in E. coli. Although the fusion lacking the putative -10 element expresses significant $\beta$-galactosidase activity in the absence of ToxR (approximately 2000 units), it is apparent that other sequences within the DNA fragment are capable of initiating transcription in E. coli. This is demonstrated when a fragment harbouring only the DNA between -172 and -117 relative to the ToxR-dependent start site was fused to lacZ (Fig. 7). This fragment lacks the complete putative promoter element yet expresses approximately 900 units of $\beta$-galactosidase activity in the absence of ToxR. Again, as this fragment does not contain the ToxR-binding region, $\beta$-galactosidase activity was unaffected by the presence of ToxR.

Neither ToxA nor ToxT directly activates a chromosomal toxT-lacZ fusion in E. coli

To determine if the inability of ToxR to directly activate the toxT-lacZ fusions in E. coli is an artefact of having the fusions expressed from plasmids, we constructed $E$. coli strain DH92 as described in the Experimental procedures. This $E$. coli strain, which has a toxT-lacZ gene fusion on the chromosome, was transformed with pVJ21 (toxRS ${ }^{+}$) or pACYC184 and assayed for $\beta$-galactosidase activity (Table 2). Although the activities were lower than those encoded by the plasmid-based fusions as a result 
of the copy number difference, the essential observation remained the same. In the absence of ToxR, strain DH92 expressed 158 units of activity and the level was reduced to 24 units by introducing ToxR. As the ctx promoter is activated by both ToxR and ToxT (DiRita et al., 1991), it was of interest to know whether ToxT has an effect on toxT-lacZ expression. To this end, we introduced plasmid pMMTT, in which toxT is cloned behind an inducible tac promoter in the vector pMMB208 (Morales et al., 1991), into the toxT-lacZ fusion strain DH92 (Table 2). pMMTT activates a tcpA-phoA fusion in $E$. coli 28-fold (unpublished data). However, pMMTT had no significant effect on $\beta$-galactosidase activity in $\mathrm{DH} 92$. From this observation it would appear that toxT expression is not directly autoregulated. When ToxR and ToxT together were tested for activation of the toxT-lacZ fusion in strain $\mathrm{DH} 92$, a similar repression effect as that seen with ToxR alone was observed (data not shown).

\section{Discussion}

Previous studies of the ToxR/ToxT system in $V$. cholerae led to a model in which virulence gene expression is controlled through a regulatory cascade (DiRita, 1992; DiRita et al., 1991; Higgins et al., 1992). According to this model, ToxR directly activates transcription of the genes coding for the cholera toxin $(c t x A B)$ and is required for the expression of toxT. The ToxT protein, an AraC-like transcriptional activator, in turn directly activates transcription of several other genes required for virulence, such as the TCP and ACF genes, as well as $c t x A B$ (DiRita et al., 1991; Higgins et al., 1992). The fact that ToxR-regulated virulence genes can be activated by supplying toxT under control of the tet or tac promoter in a tox $R$ mutant of V. cholerae (DiRita et al., 1991; P.M. Bruss, and V.J. DiRita, unpublished) indicates that regulated expression of toxT is an important step in the signal transduction pathway controlled by ToxR. Determining the precise role played by ToxR in regulating toxT expression is critical for understanding this pathway. Whether Tox $\mathrm{R}$ is directly or indirectly involved, possibly mediating its control of tox $T$ through some other activation factor, has not been explained. Our demonstration of a ToxR-regulated promoter controlling tox $T$ transcription in $V$. cholerae, and a ToxR-binding site upstream of tox $T$, imply a direct involvement by ToxR in toxT expression.

\section{ToxR-dependent expression of toxT}

It is clear from the transcriptional fusion data (Fig. 4) that the ToxR-dependent promoter identified within the tcpF-tox $T$ intergenic region is predominantly responsible for regulated expression of toxT. This conclusion is based on the approximately eightfold higher amount of $\beta$-galactosidase activity from the toxT-lacZ fusions in the tox $R^{+}$strain of $V$. cholerae, compared to the levels in the tox $R$ mutant, and the abolition of almost all activity when the -10 portion of this promoter is deleted. As is often the case for activated promoters, the -35 portion of this promoter does not conform well to the prokaryotic consensus element (Raibaud and Schwartz, 1984) and thus the presence of a binding site for a transcriptional activator directly upstream of the ToxR-dependent promoter is not surprising. The specific sequence upstream of the ToxRdependent promoter to which ToxR binds is not yet clear. In activating the $c t x$ promoter, ToxR binds a tandemly repeated heptamer, TITTGAT. This element is not present within DNA bound by ToxR upstream of toxT, and in fact this DNA does not contain any direct repeats that we can detect. The DNA between tcpF and toxT is rich in inverted repeat sequences that are predicted to form stem-loop structures (Fig. 3). It should be noted that the TIITGAT repeats upstream of $c t x$ are actually part of an element of hyphenated dyad symmetry (Mekalanos et al., 1983). Deletion of the TITTGAT repeats abolished ToxR binding (Miller et al., 1987): it is unclear whether this is the result of disrupting the direct repeats per se or of disrupting the symmetrical element composed in part by the repeats.

ToxR alone is sufficient to activate expression of a ctx-lacZ fusion in E. coli (Miller et al., 1987; Miller and Mekalanos, 1984; Ottemann et al., 1992). However, the data reported here suggest that ToxR binding is not sufficient to allow activation of a toxT-lacZ fusion in $E$. coli. The apparent repression by ToxR of toxT-lacZ fusions containing the ToxR-binding region in $E$. coli raises questions about the function of ToxR binding within this region. Clearly, ToxR is required for elevated expression of toxT in $V$. cholerae and its binding to DNA upstream of the toxT promoter suggests a direct involvement. It may simply be the case that the location of the ToxR-binding site on the toxT promoter does not allow ToxR to make the proper contact with $E$. coli RNA polymerase for activation of transcription in E. coli. This would imply that there are important differences between RNA polymerases of $E$. coli and $V$. cholerae.

It may also be that an additional factor is required for direct activation of the toxT promoter by ToxR. The nature of this putative additional requirement is only speculative at present, but candidates for it are DNA topology or other protein factors such as a subunit of the RNA polymerase or a transcriptional activator. Supercoiling and DNA topology can affect the expression of virulence genes in response to environmental stimuli (Dorman et al., 1990; Galan and Curtiss III, 1990; Hulton et al., 1990). In V. cholerae, regulated expression of the $a c f A$ and $a c f D$ gene promoters is affected by whether reporter gene fusions are chromosomally encoded or 
Table 2. Expression of a toxT-lacZ chromosomal fusion in E. coli.

\begin{tabular}{lc}
\hline Effector $^{\mathrm{a}}$ & Units of $\beta$-galactosidase \\
\hline pACYC184 (-toxRS) & 158 \\
pVJ21 (+toxRS) & 24 \\
pMMB208 $(-$ toxT) & 148 \\
pMMTT (+toxT) & 159 \\
\hline
\end{tabular}

a. See Table 3 for a description of the effector plasmids.

b. Beta-galactosidase activities are expressed as Miller units (Miller, 1972) and represent the average of a least three individual experiments.

plasmid encoded (Parsot and Mekalanos, 1992). None of the data presented in this report suggests that DNA topology plays any significant role in toxT expression. With regard to protein factors, preliminary data indicate that ToxT does not play a role in regulating its own expression, as is evident from the lack of any significant effect of ToxT on the expression of the toxT-lacZ chromosomal fusion in $E$. coli (Table 2). In different bacterial species, alternate RNA polymerase sigma factors are required for expression of regulated promoters (Akerley and Miller, 1993; Kenney and Moran, 1987; Straus et al., 1987), and perhaps a Vibrio sigma factor is required for toxT transcription. Another possibility is that a second transcriptional activator operates at the toxT promoter in conjunction with ToxR. We are currently investigating whether additional $V$. cholerae protein factors are required for the activation of toxT.

\section{Does transcriptional read-through play a role in toxT expression?}

The finding that toxT resides within the cluster of genes coding for the toxin coregulated pilus (TCP; Higgins et al., 1992; Ogierman and Manning, 1992), with many of these genes having been shown to require ToxT for expression, led us to propose that ToxR regulates toxT expression by activating transcription that initiates upstream of $t c p F$ and reads through to toxT (Higgins et al., 1992). Although the data presented here clearly indicate that there is a ToxR-dependent promoter directly upstream of toxT, they do not rule out the possibility that additional toxT transcription occurs as a result of the transcription apparatus reading through the tcpF-toxT intergenic region. Such readthrough control of a transcriptional regulator has been proposed for expression of the prfA gene of Listeria monocytogenes (Camilli et al., 1993).

Identification of a tox $T$-specific primer extension product in RNA from toxR mutant $V$. cholerae (Fig. 2) lends the best support to the hypothesis of a readthrough control mechanism as a component of toxT expression. This product may be derived from a longer transcript initiating further upstream, given that DNA upstream of the site to which it maps does not have obvious promoter elements. This ToxR-independent site is within one of the inverted repeat elements potentially forming a stem-loop structure in the RNA (Fig. 3). Secondary structures in RNA are known to be sites for RNA processing events and can function as targets for endoribonucleases (Belasco and Higgins, 1988). Perhaps the ToxR-independent primer extension product actually maps to the $5^{\prime}$ end of an RNA species generated by cleavage of a longer RNA transcript within a structure involving the inverted repeat elements. Another possibility is that secondary structure in the RNA, due to the inverted repeats, might prevent the reverse transcriptase from proceeding through and the primer extension product marks a premature termination in the extension reaction. Secondary structures have been shown to cause reverse transcriptase to pause or stop, resulting in less than full-length primer extension products (Boorstein and Craig, 1989). The speculation that premature termination of the reverse transcriptase or processing of the RNA transcript could occur at a stem-loop structure is consistent with the presence of a minor primer extension product between the ToxR-dependent and independent products on the gel shown in Fig. 2. The $3^{\prime}$ end of this product maps to a site within the inverted repeat labelled 3 in Fig. 3 . Because of the high percentage of $A$ and $T$ residues, this predicted stem-loop structure would be significantly less stable than the one directly upstream. Hence, it is expected that the role this putative structure might play in generating a primer extension product with a $3^{\prime}$ end at this position would be minor owing to its weaker predicted stability compared with the one upstream, thereby resulting in the fainter signal that was observed from this product.

Another observation supporting transcriptional readthrough as a mechanism of toxT expression is our demonstration that the terminator between tcp $F$ and tox $T$ allows downstream transcription to occur, at least when assayed in E. coli (Table 1). This result, as it stands, suggests that a small amount of readthrough transcription from upstream may generate toxT mRNA in $V$. cholerae, assuming a mechanism for readthrough transcription exists in Vibrio. Difficulties arising in measuring galactokinase activity precluded us from testing these plasmids in $V$. cholerae. We have also observed that ToxT activity in E. coli from a plasmid encoding tcpF and toxT is dependent on a plasmid promoter upstream of tcpF (Higgins et al., 1992), which suggests that readthrough does occur and can generate biologically significant levels of ToxT. A definitive demonstration of readthrough transcription of toxT in $V$. cholerae requires further experiments. Regardless of how the ToxR-independent primer extension product described in this work is generated, the role of this transcript in virulence gene regulation is questionable given that it was identified in RNA from the toxR mutant JJM43 (Fig. 2), in which the ToxR regulon is expressed poorly, if at all. 
Table 3. Strains and plasmids used in this work.

\begin{tabular}{|c|c|c|}
\hline Strain/Plasmid & Description & Reference/Source \\
\hline \multicolumn{3}{|l|}{ Strain } \\
\hline \multicolumn{3}{|l|}{ V. cholerae } \\
\hline O395 & $\mathrm{Str}^{\mathrm{P}}$ & Laboratory collection \\
\hline JJM43 & O395 $\Delta c t x A 1 \Delta$ toxR43 & Herrington et al. (1988) \\
\hline \multicolumn{3}{|l|}{ E.coli } \\
\hline K37 & galK, $\operatorname{Str}^{R}$ & NIH collection \\
\hline AAEC189 & $\begin{array}{c}\mathrm{F}^{-} \Delta \text { fim recA supE44 hsdR17 mcrA morB endA1 } \\
\text { thi-1 } \Delta \text { (argF-lac)205(U169) } \lambda^{-}\end{array}$ & Blomfield et al. (1991) \\
\hline TE2680 & $\begin{array}{l}\mathrm{F}^{-} \lambda-\mathrm{IN}(\mathrm{rmD}-\mathrm{rmE}) 1 \Delta(\mathrm{lac}) X 74 \mathrm{rps} L \text { galK2 } \\
\text { recD1903::Tn 10d-Tet trpDC700 ::putPA1303 } \\
\quad:\left[\mathrm{Kan}^{\mathrm{S}}-\mathrm{Cam}^{\mathrm{P}} \text {-lac] }\right.\end{array}$ & Elliott (1992) \\
\hline $\mathrm{DH} 92$ & TE2680 [Kan' -ptoxT-lacZ] & This work \\
\hline \multicolumn{3}{|l|}{ Plasmid } \\
\hline pKL600 & $\begin{array}{l}\text { pBR322-based transcription termination test } \\
\text { vector; } \mathrm{Ap}^{\mathrm{R}}\end{array}$ & Cheng et al. (1991) \\
\hline pB51 & $\begin{array}{l}\text { pKL600 with tcpF-toxT intergenic region insert; } \\
\mathrm{Ap}^{\mathrm{R}}\end{array}$ & This work \\
\hline ptR2 & pKL600 with $\lambda$ tR2 terminator insert; $A^{A}$ & Cheng et al. (1991) \\
\hline pTL61T & lac $Z$ transcriptional fusion vector; $A p^{R}$ & Linn and Pierre (1990) \\
\hline pACYC184 & Cloning vector; $\mathrm{Tc}^{R} \mathrm{Cam}^{\mathrm{R}}$ & $\begin{array}{l}\text { Chang and Cohen } \\
\text { (1978) }\end{array}$ \\
\hline pVJ21 & pACYC184 tc ::toxRS & Miller et al. (1989) \\
\hline pMMB208 & cloning vector; $\mathrm{Cam}^{\mathrm{R}}$ & Morales et al. (1991) \\
\hline pMMTT & pMMB208 with toxT insert & This work \\
\hline pRS551 & lac $Z$ transcriptional fusion vector; $\mathrm{Ap}^{\mathrm{R}} \mathrm{Kan}^{\mathrm{R}}$ & Simons et al. (1987) \\
\hline pRSI2 & pRS551 ptoxT-lacZ & This work \\
\hline
\end{tabular}

$\mathrm{Str}^{\mathrm{R}}$, streptomycin resistant; $\mathrm{Ap}^{\mathrm{R}}$, ampicillin resistant; $\mathrm{Tc}^{\mathrm{R}}$, tetracycline resistant; $\mathrm{Kan}^{\mathrm{R}}$, kanamycin resistant; $\mathrm{Cam}^{\mathrm{R}}$, chloramphenicol resistant.

The precise mechanism for ToxR-regulated expression of toxT remains to be elucidated, but the identification of a ToxR-regulated promoter upstream of toxT and a previously unidentified ToxR-binding site suggest a direct involvement of ToxR. The fact that the ToxR-binding sequence for toxT is completely different from that of $C t x A B$ brings up interesting questions concerning the nature of ToxR-DNA interactions. Future studies in this system will give us a clearer understanding of the precise mechanism of ToxR-regulated toxT expression and therefore of virulence regulation in $V$. cholerae, and will also provide answers to more fundamental questions of DNA-protein interactions.

\section{Experimental procedures}

\section{Bacterial strains and plasmids}

The V. cholerae and E. coli strains and plasmids used in this work are listed in Table 3. Strains were grown in LB medium (Miller, 1972) at $30^{\circ} \mathrm{C}$. Strains were maintained at $-70^{\circ} \mathrm{C}$ in LB medium plus $20 \%$ glycerol. Antibiotics were used at the following concentrations: ampicillin, $100 \mu \mathrm{g} \mathrm{ml}^{-1}$; tetracycline, $12.5 \mu \mathrm{g} \mathrm{ml}^{-1}$; kanamycin, $30 \mu \mathrm{g} \mathrm{ml}^{-1}$; chloramphenicol, $50 \mu \mathrm{g} \mathrm{ml}^{-1}$ and streptomycin, $100 \mu \mathrm{g} \mathrm{ml}^{-1}$. Transfer of plasmid DNA to $V$. cholerae was carried out by electroporation, and to $E$. coli by transformation using standard protocols (Sambrook et al., 1989).

\section{DNA manipulations}

Plasmid DNA was purified by using QIAGEN columns (QIAGEN, Inc.). PCR products containing various portions of the $t c p F$-tox $T$ intergenic region were generated using Vent $t_{B}$ DNA polymerase using the manufacturer's specified procedure (New England Biolabs). PCR reaction templates were either plasmid pDH8 (Higgins et al., 1992) containing a portion of the TCP gene cluster including the complete tcpF and toxT genes from $V$. cholerae strain 0395 , or plasmid pJM17 (Mekalanos et al., 1983) containing the ctxAB promoter region harbouring eight copies of the TIITGAT ToxR-binding sequence. When necessary, synthesized primers contained added recognition sequences for restriction endonucleases to facilitate orientation-directed cloning. PCR products were purified by agarose gel electrophoresis followed by gel extraction using the QIAEX gel extraction system (QIAGEN, Inc.). Cloning procedures were carried out using standard protocols (Sambrook et al., 1989). Doublestranded sequencing of plasmids was performed as previously reported (Higgins et al., 1992). The ctxAB promoter fragment was sequenced by using the fmol PCR sequencing kit (Promega Biotech).

\section{Primer extension}

RNA was isolated from $V$. cholerae strains by the hotphenol method (DiRita et al., 1991). Ten picamoles of primer 5'-CATTAGTTTGAAAAGATITITTCCCAATCAT-3' 
was end-labelled using $50 \mu \mathrm{Ci}$ of $\left[\gamma^{32} \mathrm{P}\right]-\mathrm{ATP}(>3000 \mathrm{Ci}$ $\mathrm{mmol}^{-1}$ ) and phage T4 polynucleotide kinase (BRL) as described (Sambrook et al., 1989). Approximately $0.2 \mathrm{pmol}$ of labelled primer was added to $20 \mu \mathrm{g}$ of RNA. Two microlitres of $5 \times$ hybridization buffer $(0.5 \mathrm{M} \mathrm{KCl}, 0.25 \mathrm{M}$ Tris $\mathrm{HCl}, \mathrm{pH} 8.3)$ and $\mathrm{dH}_{2} \mathrm{O}$ to $10 \mu \mathrm{l}$ was added. Reaction mixtures were incubated at $95^{\circ} \mathrm{C}$ for $1 \mathrm{~min}, 55^{\circ} \mathrm{C}$ for $2 \mathrm{~min}$, and then on ice for $15 \mathrm{~min}$ to allow annealing of primer to template. Five microlitres of the annealed reaction mixtures were added to tubes containing $1 \mu \mathrm{l}$ of $2.5 \mathrm{mM}$ dGTP, dATP, dTTP, and dCTP mix; $1 \mu \mathrm{l}$ of $0.1 \mathrm{M}$ dithiothreitol, $2 \mu \mathrm{l}$ of reverse transcriptase buffer $(0.25 \mathrm{M}$ Tris $\mathrm{HCl}, \mathrm{pH} 8.3,0.375 \mathrm{M} \mathrm{KCl}, 15 \mathrm{mM} \mathrm{MgCl}$, $2 \mathrm{U}$ of RNasin (Promega Biotech) per $\mu \mathrm{l}$ ), and $1 \mu \mathrm{l}(200 \mathrm{U})$ of Superscript reverse transcriptase (BRL). Reaction mixtures were incubated at $44^{\circ} \mathrm{C}$ for $45 \mathrm{~min}$, and then $5 \mu \mathrm{l}$ of Sequenase stop buffer (United States Biochemical) was added to terminate the reactions. Extended reaction mixtures were heated to $95^{\circ} \mathrm{C}$ for $3 \mathrm{~min}$ and $5 \mu \mathrm{l}$ resolved by electrophoresis in $5 \%$ denaturing polyacrylamide gels and visualized by autoradiography following standard protocols (Sambrook et al., 1989).

\section{Transcription termination analysis}

A PCR product harbouring the portion of the tcpF-tox $T$ intergenic region indicated in the legend to Fig. 3 , with flanking $\mathrm{BamHI}$ and $\mathrm{Xbal}$ restriction site sequences, was generated and subcloned into BamHI-Xbal-digested pKL600 to create pB51. After verifying the intergenic sequence to be wild type, pB51 DNA was transformed into E. coli strain K37 and galactokinase activity measured as described (Adhya and Miller, 1979) with the exception that cells were grown in LB medium without the addition of IPTG.

\section{Transcriptional fusion analysis}

PCR products harbouring various portions of the tcpF-toxT intergenic region were generated with flanking $X$ bal and BamHI sites and subcloned into Xbal-BamHI-digested pTL61T. After verifying the intergenic sequences to be wild type, plasmid DNA was transferred into $V$. cholerae strains or $E$. coli strain AAEC189. The $E$. coli strain also contained either the pACYC184 vector or pVJ21 (tox $\left.R^{+} S^{+}\right)$. V. cholerae cells harbouring plasmids were grown overnight in LB medium with a starting $\mathrm{pH}$ of 8.5 at $30^{\circ} \mathrm{C}$ then diluted $1: 50$ in fresh LB medium $\mathrm{pH} 6.5$ and grown for $3 \mathrm{~h}$ at $30^{\circ} \mathrm{C}$. Cells were harvested and $\beta$-galactosidase activity measured as previously described (Miller, 1972). There is no observable effect of $\mathrm{pH}$ on ToxR-regulated gene expression in E. coli, so assays for $E$. coli constructs were carried out as in $V$. cholerae except the media starting $\mathrm{pH}$ was always 6.5 .

\section{Preparation of membrane fractions containing Tox $R$ and ToxS}

E. coli membrane fractions containing ToxR and ToxS were prepared as previously described (Miller et al., 1987), except that strain AAEC189 harbouring the plasmid PACYC184 or pVJ21 was used as the source of the membranes. Total protein concentration was determined by reading the optical density at $280 \mathrm{~nm}\left(\mathrm{OD}_{280}\right)$ of membrane fractions.

\section{DNA gel mobility assays}

Retardation of radioactively labelled DNA fragments was performed as described previously (Miller et al., 1987; Ottemann et al., 1992) with the following modifications. Gelextracted PCR products, having been subcloned and the sequences verified, were end-labelled using $\left[\gamma_{-}{ }^{32} \mathrm{P}\right]-\mathrm{ATP}$ $\left(>3000 \mathrm{Cimmol}^{-1}\right.$ ) and phage T4 polynucleotide kinase (BRL) (Sambrook et al., 1989). Assays were performed in $20 \mu$ l volumes with membrane fractions containing a final protein concentration of $500 \mu \mathrm{g} \mathrm{ml}^{-1}$ and including $12.5 \mu \mathrm{g} \mathrm{ml}^{-1}$ sheared salmon sperm DNA. Competition assays were performed similarly with the addition of a 10-fold mass excess of unlabelled PCR-generated competitor DNA to the binding reaction.

\section{Construction of a toxT-lacZ chromosomal fusion in E. coli}

The toxT-lacZ chromosomal fusion strain DH92 was constructed using the method of Elliott (1992). Briefly, a PCR product, containing DNA from -172 to +45 relative to the ToxR-dependent toxT transcription initiation site (Fig. 3), was generated with flanking $\mathrm{ECORI}$ and $\mathrm{BamHI}$ restriction endonuclease sites. This fragment was subcloned into EcoRI-BamHI-digested pRS551 (Simons et al., 1987) yielding plasmid pRSI2. After verifying the sequence was wild type, pRSI2 DNA was linearized by digesting with Xhol. Linear DNA was transformed into $E$. coli strain TE2680 and cells then selected for kanamycin resistance $\left(\mathrm{Kan}^{\mathrm{R}}\right)$ and screened for ampicillin sensitivity $\left(\mathrm{Ap}^{\mathrm{S}}\right)$ and chloramphenicol sensitivity $\left(\mathrm{Cam}^{\mathrm{S}}\right)$, which indicate recombination of the fusion on to the chromosome. A Kan ${ }^{\mathrm{R}}, \mathrm{Cam}^{\mathrm{S}}, \mathrm{Ap}^{\mathrm{S}}$ clone was designated as strain $\mathrm{DH} 92$. Plasmids containing toxR or toxT were separately transformed into $\mathrm{DH} 92$ and $\beta$-galactosidase activity measured as for $V$. cholerae strains, with the following exception: after shifting to $\mathrm{pH} 6.5$ medium, and growing for $1.5 \mathrm{~h}$, IPTG was added to a final concentration of $0.25 \mathrm{mM}$ to cultures with the pMM series plasmids and these were allowed to grow an additional $1.5 \mathrm{~h}$ before $\beta$ galactosidase activity was measured.

\section{Acknowledgements}

The authors thank David Friedman, Michael Koomey, and Michael Imperiale for critical review of this manuscript. We also acknowledge Maria Sandkvist and Paula Bruss for helpful discussions. Appreciation is given to Karen Ottemann for technical assistance with the gel mobility-shift assays. This work was supported by Public Health Service Grants Al-31645 and RR-00200 from the National Institutes of Health and by Rackham and Phoenix faculty awards from the University of Michigan. DNA sequence analysis work was supported in part from Grant M01 RR-00042 to the Clinical Research Center at the University of Michigan. D.E.H. is a recipient of a Rackham Merit Fellowship from the University of Michigan and V.J.D. is the recipient of JFRA-392 from the American Cancer Society.

\section{References}

Adhya, S., and Miller, W. (1979) Modulation of the two 
promoters of the galactose operon of Escherichia coli. Nature 279: 492-494.

Akerley, B.J., and Miller, J.F. (1993) Flagellin gene transcription in Bordetella bronchiseptica is regulated by the BvgAS virulence control system. J Bacteriol 175: 3468-3479.

Belasco, J.G., and Higgins, C.F. (1988) Mechanisms of mRNA decay in bacteria: a perspective. Gene 72: 1523.

Blomfield, I.C., McClain, M.S., and Eisenstein, B.I. (1991) Type 1 fimbriae mutants of Escherichia coli K12: characterization of recognized afimbriate strains and construction of new fim deletion mutants. Mol Microbiol 5: 14391445.

Boorstein, W.R., and Craig, E.A. (1989) Primer extension analysis of RNA. Meth Enzymol 180: 347-369.

Camilli, A., Tilney, L.G., and Portnoy, D.A. (1993) Dual roles of plcA in Listeria monocytogenes pathogenesis. Mol Microbiol 8: 143-157.

Chang, A.C.Y., and Cohen, S.N. (1978) Construction and characterization of amplifiable multicopy DNA cloning vehicles derived from the P15A cryptic miniplasmid. $J$ Bacteriol 134: 1141-1156.

Cheng, S.-W.C., Lynch, E.C., Leason, K.R., Court, D.L., Shapiro, B.A., and Friedman, D.I. (1991) Functional importance of sequence in the stem-loop of a transcription terminator. Science 254: 1205-1207.

DiRita, V.J. (1992) Co-ordinate expression of virulence genes by ToxR in Vibrio cholerae. Mol Microbiol 6: 451-458.

DiRita, V.J., and Mekalanos, J.J. (1991) Periplasmic interaction between two membrane regulatory proteins, ToxR and ToxS, results in signal transduction and transcriptional activation. Cell 64: 29-37.

DiRita, V.J., Parsot, C., Jander, G., and Mekalanos, J.J. (1991) Regulatory cascade controls virulence in Vibrio cholerae. Proc Natl Acad Sci USA 88: 5403-5407.

Dorman, C.J., Bhriain, N.N., and Higgins, C.F. (1990) DNA supercoiling and environmental regulation of virulence gene expression in Shigella flexneri. Nature 344: 789792.

Elliott, T. (1992) A method for constructing single-copy lac fusions in Salmonella typhimurium and its application to the hemA-prfA operon. J Bacteriol 174: 245-253.

Galan, J.E., and Curtiss III, R. (1990) Expression of Salmonella typhimurium genes required for invasion is regulated by changes in DNA supercoiling. Infect Immun 58: $1879-1885$.

Herrington, D.A., Hall, R.H., Losonsky, G., Mekalanos, J.J., Taylor, R.K., and Levine, M.M. (1988) Toxin, toxincoregulated pili, and the ToxR regulon are essential for Vibrio cholerae pathogenesis in humans. J Exp Med 168: 1487-1492.

Higgins, D.E., Nazareno, E., and DiRita, V.J. (1992) The virulence gene activator ToxT from Vibrio cholerae is a member of the AraC family of transcriptional activators. $J$ Bacteriol 174: 6974-6980.

Hulton, C.S.J., Seirafi, A., Hinton, J.C.D., Sidebotham, J.M., Waddell, L., Pavitt, G.D., Owen-Hughes, T., Spassky, A., Buc, H., and Higgins, C.F. (1990) Histone-like protein $\mathrm{H} 1$ (H-NS), DNA supercoiling and gene expression in bacteria. Gene 63: 631-642.

Kaufman, M.R., Seyer, J.M., and Taylor, R.K. (1991)
Processing of TCP pilin by TcpJ typifies a common step intrinsic to a newly recognized pathway of extracellular protein secretion by gram-negative bacteria. Genes Dev 5: 1834-1846.

Kenney, T.J., and Moran, C.P., Jr. (1987) Organization and regulation of an operon that encodes a sporulationessential sigma factor in Bacillus subtilis. J Bacteriol 169: 3329-3339.

Linn, T., and Pierre, R.S. (1990) Improved vector system for constructing transcriptional fusions that ensure independent translation of lacZ. J Bacteriol 172: 10771084.

Mekalanos, J.J., Swartz, D.J., Pearson, G.D.N., Harford, N., Groyne, F., and de Wilde, M. (1983) Cholera toxin genes: nucleotide sequence, deletion analysis and vaccine development. Nature 306: 551-557.

Miller, J.H. (1972) Experiments in Molecular Genetics. Cold Spring Harbor, New York: Cold Spring Harbor Laboratory Press.

Miller, V.L., and Mekalanos, J.J. (1984) Synthesis of cholera toxin is positively regulated at the transcriptional level by ToxR. Proc Natl Acad Sci USA 81: 3471-3475.

Miller, V.L., DiRita, V.J., and Mekalanos, J.J. (1989) Identification of toxS, a regulatory gene whose product enhances ToxR-mediated activation of the cholera toxin promoter. J Bacteriol 171: 1288-1293.

Miller, V.L., Taylor, R.K., and Mekalanos, J.J. (1987) Cholera toxin transcriptional activator ToxR is a transmembrane DNA binding protein. Cell 48: 271-279.

Morales, V.M., Backman, A., and Bagdasarian, M. (1991) A series of wide-host-range low-copy-number vectors that allow direct screening for recombinants. Gene 97: 3947.

Ogierman, M.A., and Manning, P.A. (1992) Homology of $\mathrm{TcpN}$, a putative regulatory protein of Vibrio cholerae, to the AraC family of transcriptional activators. Gene 116: 9397.

Ogierman, M.A., Zabihi, S., Mourtzios, L., and Manning, P.A. (1993) Genetic organization and sequence of the promoterdistal region of the tcp gene cluster of Vibrio cholerae. Gene 126: 51-60.

Ottemann, K.M., DiRita, V.J., and Mekalanos, J.J. (1992) ToxR proteins with substitutions in residues conserved with OmpR fail to activate transcription from the cholera toxin promoter. J Bacteriol 174: 6807-6814.

Parsot, C., and Mekalanos, J.J. (1992) Structural analysis of the $a c f A$ and $a c f D$ genes of Vibrio cholerae: effects of DNA topology and transcriptional activators on expression. $J$ Bacteriol 174: 5211-5218.

Peterson, K.M., and Mekalanos, J.J. (1988) Characterization of the Vibrio cholerae ToxR regulon: identification of novel genes involved in intestinal colonization. (Published erratum appears in Infect Immun (1989) 57: 660.) Infect Immun 56: 2822-2829.

Raibaud, O., and Schwartz, M. (1984) Positive control of transcription initiation in bacteria. Annu Rev Genet 18: 173-206.

Sambrook, J., Fritsch, E.F., and Maniatis, T. (1989) Molecular Cloning: A Laboratory Manual. 2nd edn. Cold Spring Harbor, New York: Cold Spring Harbor Laboratory Press.

Simons, R.W., Houman, F., and Kleckner, N. (1987) 
Improved single and multicopy lac-based cloning vectors for protein and operon fusions. Gene 53: 85-96.

Stock, J.B., Ninfa, A., and Stock, A.M. (1989) Protein phosphorylation and regulation of adaptive responses in bacteria. Microbiol Rev 53: 450-490.

Straus, D.B., Walter, W.A., and Gross, C.A. (1987) The heat shock response of $E$. coli is regulated by changes in the concentration of sigma 32. Nature 329: 348-351.

Taylor, R.K., Miller, V.L., Furlong, D.B., and Mekalanos, J.J. (1987) Use of phoA gene fusions to identify a pilus colonization factor coordinately regulated with cholera toxin. Proc Natl Acad Sci USA 84: 2833-2837. 
This document is a scanned copy of a printed document. No warranty is given about the accuracy of the copy. Users should refer to the original published version of the material. 